\title{
PROPIEDADES PSICOMÉTRICAS DE UNA ESCALA DE CELOS ROMÁNTICOS EN ESTUDIANTES UNIVERSITARIOS
}

\section{Psychometric properties of a romantic jealousy scale} in university students

\author{
Walter L. Arias Gallegos*, Renzo Rivera** y Karla D. Ceballos Canaza*** \\ Universidad Católica San Pablo, Arequipa, Perú
}

\section{Resumen}

En la presente investigación se analizan las propiedades psicométricas de la Escala de Medición de Intensidad de Celos en la ciudad de Arequipa, para lo cual se evaluó a 212 estudiantes universitarios de una universidad privada de Arequipa Metropolitana. Los datos se procesaron a través de los programas SPSS y Factor. Los resultados indican que la prueba presenta una estructura bifactorial que alude a factores externos e internos de los celos, con adecuados indices de confiabilidad ( $\alpha=0.880$ y $\alpha=0.925$, respectivamente). Ambos factores explican el $44.1 \%$ de la varianza total de la prueba y cuentan con índices de bondad de ajuste satisfactorios en la mayoría de los casos.

Palabras clave: Celos, validez, confiabilidad, psicometría.

\begin{abstract}
In the following research it has been analyzed the psychometrical properties of the Jealousy Intensity Measure Scale in Arequipa city, so there were assessed 212 university students from a private university located in Arequipa. The data was processed through SPSS and Factor programs. The results indicate that the test presents a bifactorial structure which is related with external and internal factors about jealousy, with adequate reliability indexes $(\alpha=0.880$ and $\alpha=$ 0.925 , respectively). Both factors explain the $44.1 \%$ of the total variance of the test and present satisfactory goodness of fit indexes in the major cases.
\end{abstract}

Key words: Jealousy, validity, reliability, psychometrics

\footnotetext{
* Psicólogo.warias@ucsp.edu.pe.https://orcid.org/0000-0002-4183-5093

**Psicólogo.crrivera@ucsp.edu.pe. https://orcid.org/0000-0002-4976-9952

*** Psicóloga. karla.ceballos@ucsp.edu.pe.https://orcid.org/0000-0001-8006-3738
} 


\section{INTRODUCCIÓN}

Los celos pueden definirse como un emoción que surge ante la sospecha real o imaginaria de amenaza a una relación que se considera valiosa (García, Gómez, y Canto, 2001). Aunque los celos pueden darse en diversos contextos como en la familia (Costa, y da Silva, 2008a) o el trabajo (Girbés, y Martín, 2015), y otros escenarios sociales; han sido más estudiados en contextos relacionales de pareja. Una relación de pareja es una "relación diádica que involucra interacción social y actividades conjuntas con la implícita o explícita intención de continuar la relación hasta que una de las partes la termine o se establezca otro tipo de relación como la cohabitación o matrimonio" (Strauss, como se citó en Hernando-Gómez et al., 2016, p. 2).

Se trata entonces, de una emoción dinámica y compleja, que tienen lugar cuando se percibe que lo que uno posee lo puede perder (Reidl et al., 2005). Es dinámica en tanto puede cambiar desde los "celos normales", hasta sentimientos asociados a inseguridad y a conductas francamente patológicas de tipo delirante (Ventura-León et al., 2018). Su complejidad deviene del hecho de que comprende tanto factores emocionales, cognitivos, conductuales y fisiológicos (Granados, 2016). Fisiológicamente, por ejemplo, se ha reportado que el ritmo cardíaco y la actividad electrodérmica se elevan cuando las personas imaginan que su pareja les engaña (Buss et al., 1992). Otros estudios han reportado que los celos desencadenan $66.4 \%$ de respuestas conductuales, $58.8 \%$ de respuestas emocionales, $18.7 \%$ de respuestas cognitivas y $13.4 \%$ de respuestas fisiológicas (Carrera, y García, 1996).

En ese sentido, algunos autores valoran los celos como una experiencia negativa porque está asociada a sentimientos como duelo, desesperación, culpa, enojo, rabia y odio (Canto, García y Gómez, 2009); además, las personas que experimentan celos tienen bajos niveles de autoestima, altos niveles de neuroticismo, apego ansioso y dependencia emocional (VenturaLeón et al., 2018). Asimismo, los celos suelen ser más frecuentes en las relaciones conflictivas y tienen poder predictivo en las agresiones de pareja durante el noviazgo o en la violencia conyugal (Blázquez et al., 2009; Fernández et al., 2019; Hernando-Gómez et al., 2016). Por otro lado, los celos románticos pueden cumplir algunas funciones más positivas en la elación de pareja, pues permiten que los individuos mantengan, protejan o restauren sus lazos afectivos; actuando como un termómetro de las relaciones amorosas (Reidl, Sierra, y Fernández, 2005).

Teóricamente, los celos han sido explicados desde diversos enfoques o modelos teóricos. El psicoanálisis por ejemplo, considera los celos como un fenómeno nuclear en el complejo de Edipo que está relacionado con los impulsos eróticos y agresivos (Freud, 2001). Desde un enfoque conductual, los celos pueden ser considerados como una "conducta operante" que se refuerza negativamente por la eliminación del rival (Costa, y da Silva, 2008a), o positivamente a través de mecanismos sociales (Costa, y da Silva, 2008b). De modo que el refuerzo afecta el aumento o disminución de la conducta celosa, y una forma de abordarlo terapéuticamente es a través del análisis de los antecedentes que desencadenan la conducta celosa (Hernández-Ardieta, 2010). Desde un enfoque cognitivo, los celos serían producto de diversas creencias irracionales sobre uno mismo o la pareja, que están mediados por experiencias tempranas de vulnerabilidad psicológica, como carencias afectivas parentales. Entre los estilos de pensamiento que se han asociado con los celos se tiene la intolerancia a la incertidumbre, la necesidad de control, rigidez de pensamiento, pensamiento mágico y locus de control externo (Cuesta, 2006).

Sin embargo las teorías que más han abonado los estudios sobre los celos, son las de corte evolucionista y sociocultural (Wiederman y Rice, 1993). Las teorías evolucionistas se centran en el hecho de que los varones no pueden estar seguros de la paternidad de sus hijos, por lo que el riesgo de invertir en hijo que no es suyo es mayor, mientras que en las mujeres, intentan asegurar la manutención de sus hijos buscando una pareja que les provea de cuidados y seguridad económica (García, 2020). Estas teorías tienen bastante apoyo a través de investigaciones que sugieren que mientras los varones tienen más celos ante una infidelidad sexual las mujeres tienen más celos ante una infidelidad emocional (Apaza y Roberts, 2006; Buss, 2013; Buss et al., 1992; Canto, García y Gómez, 2009; Portilla yHenao, 2010; Retana y Sánchez, 2008; Shackelford et al., 2004). 
En ese sentido, la infidelidad tiene lugar cuando se viola el compromiso de exclusividad emocional y sexual de la pareja, pero diversos factores intervienen para que se manifiesten los celos con mayor o menor intensidad. Uno de estos factores es la tendencia infiel (Apaza y Roberts, 2006), pues muchas personas (60\% de varones y $40 \%$ de mujeres) reconocen haber seducido a alguien mientras estaban comprometidos (Figueroa et al., 2018). Por otro lado, otras investigaciones señalan que los celos pueden expresarse de forma diferenciada en determinados contextos culturales (Harris, y Christenfeld, 1996; Hupka, 1981; Buss et al., 1999), lo que da pie a la formulación de hipótesis socioculturales, pues la expresión de las emociones está también mediada por la cultura (Vygotsky, 2004); de modo que los celos adquieren su significado en situaciones interpersonales construidas socialmente (Canto, García y Gómez, 2009).

Desde esta perspectiva, se evidencian cuatro elementos: el competidor, el integrante de la relación centro de deseo, la persona que experimenta lo celos y la sociedad (Granados, 2016). Así, los celos son producto de diferentes expectativas que hombres y mujeres atribuyen al comportamiento social, que son adquiridas mediante mecanismos de aprendizaje social (Wiederman, \& Rice, 1993), de modo que el conocimiento social de las emociones tiene relevancia en las interacciones interpersonales, tanto en la categorización y reconocimiento como en la orientación a la acción (Carrera y García, 1996).

En otras palabras, dentro de un contexto cultural, los celos están mediados más que por el sexo, por el género, vale decir, el conjunto de roles sexuales que son construidos a través de la socialización (Hupka, \& Bank, 1996) y la comparación social (Salovey, \& Rodin, 1984). Dentro de este enfoque, las relaciones entre los sexos reproducen las relaciones de poder en la sociedad (Rivera, \& Díaz-Loving, 2010), que tienen injerencia en las relaciones amorosas, de modo que el sexo dominante (por lo general el varón), toma al sexo socialmente débil (por lo general la mujer) como una posesión (Romero et al., 2010). Los celos serían interpretados como estrategias de control y manipulación de la pareja (Rivera et al., 2010). En ese sentido, varones y mujeres desarrollan formas de respuesta diferenciada frente a los celos, mientras los primeros experimentan agresión e ira y las segundas, sienten depresión y tristeza (Ventura-León et al., 2018).

Desde una perspectiva social, algunos estudios indican que cuando las mujeres tienen celos presentan más respuestas fisiológicas y emocionales (Carrera y García, 1996), y que en ellas, la infidelidad sexual genera más celos, tristeza y sentimientos de inferioridad; mientras que los varones reaccionan ante la infidelidad sexual de forma más intensa y agresiva (García et al., 2001). Asimismo, otros estudios socioculturales señalan que en los hombres no hay diferencias en la expresión de los celos ante una infidelidad sexual y emocional, pero cuando la relación amorosa tiene más tiempo, sí se aprecia que los varones tienen más celos sexuales. Además, cuando la pareja convive la infidelidad emocional es más amenazante para las mujeres, pero cuando no han tenido relaciones sexuales se sintieron más amenazadas por la infidelidad sexual de sus parejas (Canto et al., 2009). Lo cierto es que tanto varones como mujeres han desarrollado estrategias para la retención de la pareja y proteger su vínculo emocional con ella (Figueroa et al., 2018).

Dentro del proceso de comparación social, las características del rival también relevantes, pues las mujeres sienten más celos cuando la rival es más atractiva y los varones cuando el rival es más dominante o posee mayor estatus social (Girbés y Martín, 2015). Ello no quiere decir que los varones no sientan celos ante las características físicas del rival, pues en un estudio realizado con sujetos mexicanos se encontró que los varones sienten más molestia cuando el rival es más alto, tiene espalda ancha y mayor musculatura (González et al., 2013). Asimismo, en otro estudio se reportó que las mujeres valoran más que lo físico, el carácter agradable, la inteligencia, la sinceridad y la fidelidad sexual de sus parejas (Figueroa et al., 2018).

Todos estos datos aportan evidencias a favor de las teorías socioculturales, tomando como fenómenos explicativos la dominancia social del varón a través de los roles sexuales aprendidos y los procesos de comparación social. Para el caso de los jóvenes y adolescentes, los celos se asocian con conductas sexistas y violencia en las relaciones de pareja durante 
el noviazgo (Fernández et al., 2019). También se ha reportado que los celos se relacionan con el amor de tipo adictivo, y cuando la persona es temerosa tiende a tener más desconfianza en sus relaciones de pareja (Retana y Sánchez, 2008). Otros estudios señalan que los celos se relacionan con el afrontamiento (Carrera y García, 1996), y que los estilos de afrontamiento difieren en tanto si los celos son relacionales o románticos (Reidl et al., 2005).

Asimismo, un desencadenante de los celos en las relaciones de pareja en estudiantes universitarios suele ser la detección de mentiras en la pareja (Yañez et al., 2020), y se ha señalado también que la dependencia emocional se asocia con los celos (De la Villa et al., 2018). De hecho, la prevalencia de celos entre los jóvenes suele ser preocupante, por ejemplo, un estudio con estudiantes universitarios mexicanos reportó que el $60 \%$ tenía un nivel moderado de celos, $15 \%$ niveles altos y 25\% niveles bajos; además, de manifestar sentimientos de enojo, dolor, desconfianza, intriga y egoísmo (Granados, 2016).

En Arequipa, se ha reportado que mientras el $42 \%$ de estudiantes universitarios varones presentan niveles moderados de celos, el $33 \%$ de mujeres presenta niveles moderados de celos; pero el $8 \%$ de mujeres y el $5 \%$ de varones tenían niveles severos de celos. Además, las mujeres tuvieron más celos frente a una infidelidad emocional y los varones frente a una infidelidad sexual (Apaza y Roberts, 2006). Otro estudio reportó que en 973 estudiantes universitarios, los celos están asociados con los vínculos de control sin afecto brindados por los padres (Martínez, 2009). Asimismo, en otra investigación con 630 estudiantes universitarios, se reportó que en los estudiantes varones los factores más predominantes para la infidelidad fueron el no estar enamorado, la falta de estabilidad económica, insatisfacción en la intimidad sexual y poca comunicación; mientras que en las mujeres, los factores más predominantes para ser infieles fueron la falta de estabilidad económica, el no estar enamorada, poca comunicación y la falta de confianza y comprensión con la pareja (Arenas y Cárdenas, 2009).

Más recientemente, se ha encontrado que el nivel de compromiso (como dimensión de la teoría triarquica del amor de Sternberg) se relaciona significativamente con los celos (Juárez, \& Pacheco, 2018). Como puede verse, los celos han sido objeto de estudio en diversas investigaciones en esta ciudad, pero no se han reportado investigaciones que valoren la validez y la confiabilidad de pruebas que evalúan esta variable, lo cual constituye un problema que amerita ser abordado instrumentalmente. Por ello, el presente estudio pretende estimar las propiedades psicométricas de la Escala de Medición de Intensidad de Celos (Apaza y Roberts, 2006).

En ese sentido, la evaluación de los celos se ha hecho mediante situaciones imaginarias de infidelidad, por medio de la técnica de incidentes críticos o a través de recuerdos autodefinitorios (Reidl et al., 2005); que dan origen a diversas pruebas psicológicas.Por ejemplo, Buss et al. (1992) desarrollaron el Cuestionario de la Diferencia de Celos según el Género que plantea seis dilemas de opción forzada entre dos alternativas de respuesta para cada caso. También se tiene la Escala de Celos Románticos de White, que presenta una confiabilidad de 0.89 obtenida mediante la prueba alfa de Cronbach, que evidencia valores diferenciados para varones y mujeres (Montes-Berges, 2008). Otra prueba psicológica es la Interpersonal Jealousy Scale que consta 28 ítems en una escala de respuesta tipo Likert de nueve niveles, que obtuvo una estructura unidimensional un índice de confiabilidad de 0.90 tras ser aplicada a 600 adultos colombianos (MartínezLeón et al., 2018).

Precisamente, a nivel de Latinoamérica, en México, Díaz-Loving et al. (1989) crearon el Inventario Multimodal de Celos con buenos índices de validez y confiabilidad, que ha sido empleado en algunas investigaciones locales (Juárez y Pacheco, 2018). También se tiene la Escala para estimar las Razones por las que Somos Infieles que consta de 23 ítems en una escala de respuesta tipo Likert, distribuidos en cinco factores que explican el $61.51 \%$ de la varianza total. Las dimensiones de esta prueba son: Insatisfacción con la relación, Sexualidad, Legitimación de la infidelidad, Apertura sociosexual, Constancia y estabilidad emocional; y cuentan con adecuados índices de confiabilidad (Figueroa et al., 2018). 
En Chile se ha validado el Inventario de Comportamientos de la Pareja que provocan Celos, que fue creada en Holanda, y cuenta con 38 ítems en una escala tipo Likert con cinco niveles de respuesta, que están distribuidos en cuatro dimensiones: Comportamiento infiel, Comportamiento sospechoso, Pornografía e Inversión tecnológica. La prueba resultó confirmar solo tres de las dimensiones originales y se calculó la confiabilidad mediante el método testretest, con índices superiores a 0.7 para cada uno de los factores (Fernández et al., 2014). En México, se construyó el Inventario Multidimensional de Celos Románticos que consta de 19 ítems y cinco factores (Conceptual, Circunstancias, Afectiva, Cognitiva y Conductual) que tienen índices de confiabilidad superiores a 0.8 en cada escala (Mota et al, 2016).

Finalmente, el Inventario de Comunicación Emocional en las Relaciones Románticas es una escala creada originalmente por Sánchez (2012) en México, que cuenta con ocho factores que explican el $49.3 \%$ de la varianza total. Estas dimensiones son: Amor-felicidad, Pasión, Celos, Tristeza, Enojo, Sorpresa positiva y Sorpresa negativa. Esta escala ha sido validada y analizada psicométricamente en Perú por Ventura-León et al. (2018), quienes reportaron que el análisis de invarianza factorial aplicado, no mostró diferencias significativas entre varones y mujeres, y su tamaño de efecto fue inexistente.

\section{Método}

Dado que se trata de analizar las propiedades psicométricas de una prueba que evalúa la intensidad de celos, el tipo de investigación es instrumental (Montero y León, 2002).

\section{Muestra}

La muestra está conformada por 212 estudiantes universitarios de una universidad privada de la ciudad de Arequipa que fueron seleccionados de forma no probabilística, mediante la técnica de grupos intactos. El $78.6 \%$ fueron mujeres y el $21.4 \%$ varones, con una edad de 18 a 21 años y una media de 19 años, quienes aceptaron participar voluntariamente del estudio luego de que se les fueron explicados los fines de la investigación.

\section{Instrumento}

Se aplicó la Escala de Medición de Intensidad de Celos (Apaza y Roberts, 2006) que consta de 29 ítems con una escala de respuesta tipo Likert de cinco alternativas de respuesta, desde Siempre (4) a Nunca (0). La prueba dura aproximadamente 20 minutos en ser aplicada y ofrece cuatro niveles de celos: Leve, Moderado, Severo y Excesivo. Cuenta además con valores de validez y confiabilidad adecuados con una estructura unidimensional y un índice superior al 0.7 calculado con la prueba alfa de Cronbach.

\section{Procedimiento}

Primeramente se solicitaron los permisos correspondientes y se coordinó la aplicación del instrumento con el profesor, pues la recolección de datos se realizó durante las horas de clases, de forma colectiva. Los estudiantes provienen de cuatro clases y se consideró solo a los que tenían mayoría de edad y los que decidieron participar voluntariamente del estudio, luego de que se les explicó la finalidad de la investigación y que firmaron el consentimiento informado. Los datos fueron recogidos en el segundo semestre del año 2018, entre los meses de setiembre y octubre.

\section{Análisis de datos}

Los datos se procesaron estadísticamente siguiendo los criterios de la teoría clásica de los test y la teoría factorial (Kaplan y Saccuzzo, 2006). En primer lugar, se calcularon los estadísticos descriptivos de los ítems (media, intervalo de confianza, varianza, asimetría y curtosis), se realizaron correlaciones ítem-test y el análisis factorial exploratorio mediante dos softwares: el paquete estadístico SPSS versión 20 (IBM, 2011) y el programa Factor versión 10.5.03 (Lorenzo-Seva, \& Ferranzo, 2017). El cálculo de la confiabilidad se realizó mediante el método de consistencia interna con la prueba Alfa de Cronbach (Livia y Ortiz, 2014).

\section{RESULTADOS}

En la Tabla 1 se muestran los estadísticos descriptivos de los 29 ítems de la Escala de Medición de Intensidad de Celos donde se observan los siguientes valores: media, intervalos de confianza, varianza, asimetría, curtosis y las correlaciones ítem-test corregidas. Como se puede apreciar, los valores tienen 
medias que van de 0.962 para el ítem 19 y 2.104 para el ítem 1 . Asimismo, los valores de asimetría y curtosis se ubican dentro del rango de $-1.5 / 1.5$, que indican una tendencia a la normalidad (Pérez y Medrano, 2010), salvo en el ítem 5 que presenta una curtosis elevada fuera de este rango. En cuanto a las correlaciones ítem-test se tiene que la mayoría de ítems presentan coeficientes superiores a 0.2 (Kline, 1995).

Tabla 1.

Estadísticos descriptivos

\begin{tabular}{lcccccc}
\hline $\mathrm{N}^{\circ}$ Ítem & Media & IC 95\% & Varianza & Asimetría & Curtosis & citc \\
\hline Ítem 1 & 2.104 & $1.84-2.37$ & 1.131 & 0.028 & -0.827 & 0.312 \\
Ítem 2 & 1.557 & $1.30-1.82$ & 1.096 & 0.322 & -0.489 & 0.473 \\
Ítem 3 & 1.849 & $1.56-2.14$ & 1.317 & -0.003 & -1.009 & 0.318 \\
Ítem 4 & 1.736 & $1.42-2.05$ & 1.553 & 0.218 & -0.893 & 0.347 \\
Ítem 5 & 1.632 & $1.34-1.92$ & 1.365 & 0.466 & -0.493 & 0.416 \\
Ítem 6 & 1.962 & $1.68-2.25$ & 1.319 & -0.152 & -0.816 & 0.434 \\
Ítem 7 & 1.811 & $1.57-2.06$ & 0.964 & 0.147 & -0.552 & 0.512 \\
Ítem 8 & 1.387 & $1.12-1.66$ & 1.162 & 0.319 & -0.988 & 0.554 \\
Ítem 9 & 1.509 & $1.21-1.81$ & 1.439 & 0.392 & -0.759 & 0.583 \\
Ítem 10 & 1.340 & $1.04-1.64$ & 1.470 & 0.477 & -0.840 & 0.590 \\
Ítem 11 & 1.934 & $1.62-2.25$ & 1.571 & 0.126 & -1.050 & 0.418 \\
Ítem 12 & 1.198 & $0.90-1.49$ & 1.385 & 0.730 & -0.413 & 0.636 \\
Ítem 13 & 1.783 & $1.48-2.09$ & 1.472 & 0.137 & -0.965 & 0.478 \\
Ítem 14 & 1.179 & $0.91-1.45$ & 1.147 & 0.612 & -0.331 & 0.664 \\
Ítem 15 & 1.575 & $1.28-1.87$ & 1.395 & 0.321 & -0.878 & 0.459 \\
Ítem 16 & 1.594 & $1.31-1.88$ & 1.298 & 0.422 & -0.475 & 0.568 \\
Ítem 17 & 1.217 & $0.93-1.51$ & 1.340 & 0.672 & -0.391 & 0.606 \\
Ítem 18 & 1.160 & $0.85-1.47$ & 1.512 & 0.858 & -0.372 & 0.710 \\
Ítem 19 & 0.962 & $0.68-1.25$ & 1.300 & 0.961 & -0.143 & 0.629 \\
Ítem 20 & 1.792 & $1.52-2.06$ & 1.164 & 0.150 & -0.694 & 0.553 \\
Ítem 21 & 1.755 & $1.47-2.04$ & 1.336 & 0.381 & -0.561 & 0.588 \\
Ítem 22 & 1.840 & $1.57-2.11$ & 1.172 & 0.189 & -0.561 & 0.234 \\
Ítem 23 & 1.566 & $1.26-1.87$ & 1.453 & 0.333 & -0.860 & 0.301 \\
Ítem 24 & 1.415 & $1.11-1.72$ & 1.507 & 0.492 & -0.756 & 0.267 \\
Ítem 25 & 1.396 & $1.09-1.70$ & 1.485 & 0.497 & -0.798 & 0.331 \\
Ítem 26 & 1.717 & $1.43-2.01$ & 1.354 & 0.280 & -0.804 & 0.357 \\
Ítem 27 & 1.264 & $0.98-1.55$ & 1.270 & 0.542 & -0.524 & 0.336 \\
Ítem 28 & 1.679 & $1.35-2.00$ & 1.690 & 0.303 & -0.999 & 0.346 \\
Ítem 29 & 1.245 & $0.96-1.53$ & 1.279 & 0.728 & -0.230 & 0.436 \\
\hline & & & & &
\end{tabular}

Nota: $\mathrm{IC}=$ Intervalo de Confianza; citc= correlación ítem-test 
Posteriormente se procedió a calcular el análisis factorial exploratorio mediante de extracción de mínimos cuadrados no ponderados robustos y el procedimiento el procedimiento de óptima implementación de análisis paralelo (Timmerman y Lorenzo-Seva, 2011) para la determinación del número de factores de la prueba, y teniendo en cuenta la cantidad de la muestra se utilizó el sesgo corregido y acelerado como técnica de análisis robusto (Lambert et al., 1991), mientras que para la extracción de los factores se aplicó el método de mínimos cuadrados no ponderados robustos (RULS) con rotación Varimax RAW. El cálculo de la adecuación muestral obtuvo un valor KMO de 0.815 y un nivel alto de significancia en la prueba de esfericidad de Bartlett $\left(\mathrm{x}^{2}=1366.2\right.$; $\mathrm{gl}=$ 406; $p=0.000$ ), por tanto, se realizó el análisis factorial exploratorio que reportó dos factores que explicaron el $27.8 \%$ y $16.3 \%$ de la varianza total de la prueba, respectivamente.

Los ítems del 20 al 29 se obtuvieron saturaciones superiores a 0.450 en el Factor 1 y los ítems del 1 al 19 obtuvieron saturaciones superiores a 0.350 en el Factor 2. El cálculo de la confiabilidad de los dos factores se realizó mediante el método de consistencia interna con la prueba Alfa de Cronbach, obteniéndose índices de $\alpha=0.880$ y $\alpha=0.925$, respectivamente (ver Tabla 2). Asimismo, se obtuvo una correlación interfactorial moderada y significativa de 0.398 , que sugiere que ambos factores están relacionados en tanto que miden el mismo constructo (Lloret-Segura et al., 2014).
Tabla 2

Matriz de estructura

\begin{tabular}{|c|c|c|}
\hline Ítems & Factor 1 & Factor 2 \\
\hline Ítem 1 & & 0.350 \\
\hline Ítem 2 & & 0.531 \\
\hline Ítem 3 & & 0.346 \\
\hline Ítem 4 & & 0.446 \\
\hline Ítem 5 & & 0.460 \\
\hline Ítem 6 & & 0.448 \\
\hline Ítem 7 & & 0.582 \\
\hline Ítem 8 & & 0.617 \\
\hline Ítem 9 & & 0.617 \\
\hline Ítem 10 & & 0.692 \\
\hline Ítem 11 & & 0.479 \\
\hline Ítem 12 & & 0.721 \\
\hline Ítem 13 & & 0.561 \\
\hline Ítem 14 & & 0.761 \\
\hline Ítem 15 & & 0.503 \\
\hline Ítem 16 & & 0.585 \\
\hline Ítem 17 & & 0.689 \\
\hline Ítem 18 & & 0.771 \\
\hline Ítem 19 & & 0.783 \\
\hline Ítem 20 & 0.461 & \\
\hline Ítem 21 & 0.470 & \\
\hline Ítem 22 & 0.533 & \\
\hline Ítem 23 & 0.646 & \\
\hline Ítem 24 & 0.709 & \\
\hline Ítem 25 & 0.711 & \\
\hline Ítem 26 & 0.571 & \\
\hline Ítem 27 & 0.712 & \\
\hline Ítem 28 & 0.674 & \\
\hline Ítem 29 & 0.715 & \\
\hline$\alpha$ & 0.880 & 0.925 \\
\hline Factor 1 & 1 & 0.398 \\
\hline Factor 2 & & 1 \\
\hline
\end{tabular}

Nota: $\alpha=$ alfa de Cronbach

Finalmente, los índices de bondad de ajuste obtenidos en el procesamiento estadístico, satisfacen los criterios establecidos en la mayoría de los casos $\left(\chi^{2}=292.920 ; \mathrm{gl}=349 ; \mathrm{p}<0.986 ; \chi^{2} / \mathrm{gl}=1.343 ; \mathrm{CFI}=\right.$ 1.019 IC $95 \%$ [1.009, 1.009]; GFI= 0.961 IC $95 \%$ [0.961, 0.970]; AGFI= 0.955 IC 95\% [0.955, 0.965]; RMSEA $=0.000$ IC 95\% [0.000; 0.021]). Por lo tanto, se puede concluir que la prueba es válida y confiable. 


\section{DISCUSIÓN}

Los celos son una respuesta esperable y no necesariamente ajena a las relaciones de pareja, sobre todo si existen sospechas razonables para una infidelidad (Fernández et al., 2014). En ese sentido, los celos románticos parten de la noción de posesión, inseguridad y temor a la pérdida de la pareja, cuando hay un rival real o imaginario (Retana y Sánchez, 2008); pero los celos patológicos involucran respuestas exageradas ante amenazas reales o imaginarias, aunque son más frecuentes en el segundo caso (Ventura-León et al., 2018).

Asimismo, se ha reportado que las mujeres son más celosas que los varones, esgrimiéndose explicaciones evolucionistas y socioculturales, con mayor peso científico basado en evidencias (Wiederman y Rice, 1993). Pero también se ha reportado que los varones son más infieles que las mujeres, con porcentajes del 85\% y $60 \%$, respectivamente (Arenas y Cárdenas, 2009). Ante ello, se ha señalado que los hombres celosos no se comprometen cuando perciben riesgos y experimentan displacer, mientras que las mujeres celosas tienden a tener un amor adictivo, baja autoestima y apego inseguro (Retana y Sánchez, 2008).

El problema de los celos es muy importante, en términos de salud mental, porque se han asociado a la violencia doméstica (Fernández et al., 2019). De hecho, en el Perú los celos son la segunda causa de feminicidio en el $39.3 \%$ de los casos, y la primera causa de delito con un $46 \%$ de incidencia según los registros policiales (Ventura-León et al., 2018).

En Arequipa, los celos han sido materia de estudio de diversas investigaciones, reportándose que el $42 \%$ de los estudiantes universitarios varones presentan celos moderados y el $33 \%$ de estudiantes universitarias mujeres presenta niveles moderados de celos; siendo ligeramente mayores los celos intensos en las mujeres en comparación con los estudiantes varones, y asociarse con la infidelidad emocional en las primeras, y con la infidelidad sexual con los segundos (Apaza y Roberts, 2006).

Apesar de ello, en los estudios previos realizados en Arequipa sobre celos (Apaza y Roberts, 2006; Juárez y Pacheco, 2018; Martínez, 2009), no se ha dado cuenta de las propiedades psicométricas de instrumentos empleados; por ello, la presente investigación tuvo como objetivo la estimación de la validez y la confiabilidad de la Escala de Medición de Intensidad de Celos, que ha sido empleada en Arequipa. Nuestros resultados indican que dicho instrumento, es válido al presentar una estructura bifactorial, que implica en el primer factor (ítems del 20 al 29) aspectos externos a los celos y en el segundo factor (ítems del 1 al 19), aspectos internos de la persona que experimenta los celos. El primer factor alude a aspectos tales como el contacto con otras personas, el atractivo de la pareja, la presencia de un rival o personas interesadas en la pareja, etc.; mientras que el segundo factor se refiere a los sentimientos de posesión de la pareja, el pensamiento obsesivo, el deseo de estar cerca de la pareja, las ideas de infidelidad, los sentimientos de inseguridad en la relación y los celos propiamente dichos.

Otros datos que apoyan la estructura bifactorial de esta prueba es que se obtuvo un valor KMO alto $(0.815)$ y el porcentaje de la varianza explicada por ambos factores fue de $44.1 \%$ (Burga, 2006). Asimismo, ambos factores obtuvieron una correlación moderada $(\mathrm{r}=0.398)$, con elevados índices de confiabilidad de 0.880 y 0.925 , para el primer y segundo factor, respectivamente; que fueron calculados mediante el método de consistencia interna. Todo ello, indica que la prueba es válida y confiable (Livia y Ortiz, 2014), y que por tanto puede ser usada en investigaciones que tienen como variable objetivo a los celos. Además, los índices de bondad de ajuste calculados, fueron satisfactorios en la mayoría de los casos.

Sin embargo, debido a que la muestra no es muy grande y proviene de una sola universidad de la ciudad de Arequipa, es necesario realizar más investigaciones para valorar otras propiedades psicométricas de la escala mediante el análisis factorial confirmatorio o el análisis de invarianza, que constituyen métodos más rigurosos y precisos de análisis estadístico y psicométrico. Por otro lado, sería conveniente trabajar con muestras más amplias y representativas para obtener una versión estandarizada del instrumento. A pesar de estas limitaciones, se puede concluir que la Escala de Medición de Intensidad de Celos es válida y confiable, y que puede ser empleada con fines científicos o de evaluación psicológica, en estudiantes universitarios de nuestra ciudad. 


\section{REFERENCIAS}

Apaza, R. y Roberts, S. (2006). Celos y tendencia infiel. Revista de Psicología (Universidad Católica de Santa María), 3, 66-79.

Arenas, K. A. y Cárdenas, E. (2009). Factores relacionados con la infidelidad en la pareja según género. Revista de Psicología (Universidad Católica de Santa María), 6, 58-75.

Blázquez, M., Moreno, J. M. y García-Baamonde, M. E. (2009). Inteligencia emocional como alternativa para la prevención del maltrato psicológico en la pareja. Anales de Psicología, 25(2), 250-260.

Burga, A. (2006). La unidimensionalidad de un instrumento de medición: perspectiva factorial. Revista de Psicología de la Pontificia Universidad Católica del Perú, 24(1), 53-80.

Buss, D. M. (2013). Sexual jealously. Psychological Topics, 22(2), 155-182.

Buss, D., Larsen, R., Western, D., \& Semmetroth, J. (1992). Sex differences in jealousy: evolution, physiology and psychology. Psychological Science, 3, 251-255.

Buss, D., Shackelford, T. K., Kirkpatrick, L. A., Choe, J. C., Lim, H. K., Hasegawa, M., Hasegawa, T., \& Bennett, K. (1999). Jealousy and the nature of beliefs about infidelity: Test of competing hypotheses about sex differences in the United States, Korea and Japan. Personal Relationships, 6, 125-150.

Canto, J. M., García, P. y Gómez, L. (2009). Celos y emociones: Factores de la relación de pareja en la reacción ante la infidelidad. Athenea Digital, 15, 39-55.

Carrera, P. y García, L. (1996). Conocimiento social de los celos. Psicothema, 8(3), 445-456.

Costa, N. y da Silva, R. (2008a). Celos: un ejercicio de interpretación desde la perspectiva del análisis de la conducta. Diversitas. Perspectivas en Psicología, 4(1), 139-147.

Costa, N. y da Silva, R. (2008b). Celos: Test de definición y una hipótesis sobre la diferencia de género bajo la óptica del análisis de la conducta. Terapia Psicológica, 26(1), 15-25.
Cuesta, M. T. (2006). Intervención cognitiva en un caso de celotipia. Acción Psicológica, 4(1), 71-82.

De la Villa, M., Sirvent, C., Ovejero, A. y Cuetos, G. (2018). Dependencia emocional en las relaciones de pareja como síndrome de Artemisa: modelo explicativo. Terapia Psicológica, 36(3), 156-166.

Díaz-Loving, R., Rivera, S. y Flores, M. M. (1989). Desarrollo y análisis psicométrico de una medida multimodal de celos. Revista Mexicana de Psicología, 8(2), 111-119.

Fernández, A. M. Pavez, P. y Dufey, M. (2014). Evaluación chilena del Inventario de Comportamientos de la Pareja que provocan Celos. Revista de Psicología (Universidad de Chile), 23(2), 45-55. http://dx.doi.org/10.5354/07190581.2014 .36147

Fernández, S. Alvarado, M. y Arias, W. L. (2019). Sexismo ambivalente y violencia en las relaciones de enamoramiento en universitarios de Arequipa. Revista de Psicología (Universidad Católica San Pablo), 9(1), 47-73.

Figueroa, O., Fernández, A. M. y Jara, C. (2018). ¿Por qué somos infieles? Aplicación inicial de una escala para estimar las razones por las que los hombres y mujeres son infieles. Revista Iberoamericana de Diagnóstico y Evaluación, 48(3), 19-27. http:// dx.doi.org/10.21865/RIDEP48.3.02

Freud, S. (2001). Obras completas. Epublibre.

García, J. E. (2020). La psicología evolucionista de la familia. En W. L. Arias (Ed.), Psicología y familia . Cinco enfoques de familia y sus implicancias (pp. 89-159). Adrus.

García, P., Gómez, L. y Canto, J. M. (2001). Reacción de celos ante una infidelidad: diferencias entre hombres y mujeres y características del rival. Psicothema, 13(4), 611-616.

Girbés, B. y Martín, R. (2015). Celos y envidia en el trabajo: una revisión de los últimos 20 años. Apuntes de Psicología, 33(3), 127-136.

González, I., Armenta, C., Díaz, P. E., \& Bravo, A. (2013). Tengo celos: Percepción de las características del rival en dos contextos mexicanos. Revista de Psicología Social y Personalidad, 30(2), 50-58. 
Granados, J. A. (2016). Los celos en estudiantes universitarios: un estudio comparativo. Revista Xihmai, 11(22), 89-110.

Harris, C., \& Christenfeld, N. (1996). Jealousy and rational responses to infidelity across gender and culture. Psychological Science, 7, 378-379.

Hupka, R. (1981). Cultural determinants of jealousy. Alternative Lifestyles, 4, 310-356.

Hupka, R., \& Bank, A. (1996). Sex differences in jealousy: Evolution or social construction? Cross Cultural Research, 30, 24-59.

Hernández-Ardieta, M. L. (2010). Abordaje de una celotipia desde una perspectiva conductual. Revista de la Asociación Española de Neuropsiquiatría, 30(106), 313-317.

Hernando-Gómez, A., Maraver-López, P., \& Pazos-Gómez, M. (2016). Experiencias positivas y negativas en relaciones de pareja de jóvenes y adolescentes. Revista de Psicología (Universidad de Chile), 25(2), 1-19. http://dx.doi. org/10.5354/0719-0581.2016.44745

IBM. (2011). IBM SPSS Statistics (Version 20) [programa informático en CD-ROM].

Juárez, S. Y., \& Pacheco,A. (2018). Celos y compromiso en mujeres y hombres casados de la ciudad de Arequipa. Revista de Psicología (Universidad Católica de Santa María), 15, 63-72.

Kaplan, R. M. y Saccuzzo, D. P. (2006). Pruebas psicológicas. Principios, aplicaciones y temas. Thomson.

Kline, P. (1995). The handbook of psychological testing. Routledge.

Lambert, Z. V., Wildt, A. R., \& Durand, R.M. (1991). Approximating confidence intervals for factor loadings. Multivariate Behavioral Research, 26(3), 421-434.

Livia, J. y Ortiz, M. (2014). Construcción de pruebas psicométricas: Aplicaciones a las ciencias sociales y de la salud. Universidad Nacional Federico Villarreal.

Lloret-Segura, S., Ferreres-Traver, A., HernándezBaeza, A. y Tomás-Marco, I. (2014). El análisis factorial exploratorio de los ítems: una guía práctica, revisada y actualizada. Anales de Psicología, 30(3), 1151-1169.

Lorenzo-Seva, U. y Ferranzo, P. (2017). Factor (Version 10.5.03). Universidad Rovira i Virgili. http://psico.fcep.urv.es/utilitats/factor/

Martínez-León, N. C., Mathes, E., Avendaño, B. L., Peña, J. J., \& Sierra, J. C. (2018). Psychometric study of the Interpersonal Jealousy Scale in Colombian samples. Revista Latinoamericana de Psicología, 50(1), 21-30. http://dx.doi. org/10.14349/rlp.2018.v50.n1.3

Martínez, M. I. (2009). Vínculos parentales y celos hacia la pareja en estudiantes universitarios. Revista de Psicología (Universidad Católica de Santa María), 6, 33-39.

Montero, I. y León, O. G. (2002). Clasificación y descripción de las metodologías de investigación en Psicología. Revista Internacional de Psicología Clínica y de la Salud, 2(3), 503-208.

Mota, J., González-Arratia, N. I., Valdez, J. L., González, S. y Hernández, G. (2016). Construcción y análisis estadístico del Inventario Multidimensional de Celos Románticos: Estudio preliminar. Actualidades en Psicología, 20(120), 31-45. http://dx.doi.org/10.15517/ ap.v30i120.18977

Pérez, E. y Medrano, L. (2010). Análisis factorial exploratorio: Bases conceptuales y metodológicas. Revista Argentina de Ciencias del Comportamiento, 2(1), 58-66.

Portilla, L. Y. y Henao, G. C. (2010). Diferencias sexuales en la experiencia subjetiva de los celos: una mirada desde la Psicología Evolucionista. Pensamiento Psicológico, 8(15), 53-62.

Reidl,L., Sierra, G. y Fernández,H.(2005). Diferencias entre celos románticos y celos relacionales. Revista Iberoamericana de Diagnóstico y Evaluación, 20(2), 133-148.

Retana, B. E. y Sánchez, R. (2008). El papel de los estilos de apego y los celos en la asociación con el amor adictivo. Psicología Iberoamericana, 16(1), 15-22.

Rivera, S. y Díaz-Loving, R. (2010). Estrategias de poder en la relación de pareja. En R. Díaz-Loving 
y S. Rivera (Eds.), Antología psicosocial de la pareja. Clásicos y contemporáneos (pp. 183-213). Universidad Nacional Autónoma de México.

Rivera, S., Díaz-Loving, R., García, M., Montero, N. y Villanueva, G. B. T. (2010). Poder, control y manipulación en la pareja: algunas variantes. En R. Díaz-Loving y S. Rivera (Eds.), Antología psicosocial de la pareja. Clásicos y contemporáneos (pp. 689-735). Universidad Nacional Autónoma de México.

Salovey, P., \& Rodin, J. (1984). Some antecedents and consequences of social-comparison jealousy. Journal of Personality and Social Psychology, 47, 780-792.

Sánchez, R. (2012). Comunicación Emocional en las Relaciones Románticas: Diseño y validación de una medida en México. Interpersona. An International Journal on Personal Relationships, 6(2), 211-226. http://dx.doi.org/10.5964/ijpr. v6i2.102

Shackelford, T. K., Voracek, M., Schmitt, D. P., Buss, D. M., Weekes-Shackelford, V.A., \& Michalski, R. L. (2004). Romantic jealously in early adulthood and in later life. Human Nature, 15(3), 283-300.

Timmerman, M. E., \& Lorenzo-Seva, U. (2011). Dimensionality Assessment of Ordered
Polytomous Items with Parallel Analysis. Psychological Methods, 16, 209-220.

Ventura-León, J., Caycho-Rodríguez, T., BarbozaPalomino, M., Aparco, V., Rodas, N. (2018). Evidencias de validez e invarianza factorial de una escala breve de celos en estudiantes universitarios peruanos. Propósitos y Representaciones, 6(2). http://dx.doi.org/10.20511/pyr2018.v6n2.216

Vygotsky, L. S. (2004). Teoría de las emociones. Estudio histórico-psicológico. Akal Ediciones.

Wiederman, M. W., \& Rice, E. (1993). Gender differences in sexual jealousy: adaptionist or social learning explanation. Ethology and Sociobiology, 14, 115-140.

Yañez, G., Yañez, H. y Ferrel, F. R. (2020). Análisis de las propiedades psicométricas de un cuestionario para medir la intensidad de la molestia entre la mentira en la relación de parejas juveniles mediante el modelo Rasch. Revista de Psicología (Pontificia Universidad Católica del Perú), 38(1), 9-30. http://dx.doi.org/10.18800/ psico.202001.001

Fecha de recepción: 17 de febrero 2021

Fecha de aceptación: 24 de marzo 2021 